\title{
Silurian Palynomorphs
}

\author{
P. J. HILL ${ }^{1}$, F. PARIS ${ }^{2}$ \& J. B. RICHARDSON ${ }^{3}$ \\ ${ }^{1}$ Dept. of Geology, Derbyshire College of Higher Education, Kedleston Road, Derby DE3 1GB, U.K. \\ ${ }^{2}$ Laboratoire de Paléontologie et de Stratigraphie, Université de Rennes, 35042 Rennes Cedex, France. \\ ${ }^{3}$ Dept. of Palaeontology, British Museum (Natural History), Cromwell Road, London SW7 5BD, U.K.
}

The data obtained from an independent study of acritarchs, chitinozoans and miospores enables the determination of maximum age ranges of samples taken from three wells. These are shown in Fig. 8.

\section{ACRITARCHS}

At present, samples have been examined from six wells-A1-46, Core 2; E1-81, Cores 3, 4; F1-46, Core 3; D-31, Core 1; A1-81, Core 3 and C1-31, Core 8. The last mentioned is considered no further here as all samples have to date proved barren. The oldest of the acritarch assemblages are recorded from E1-81, Cores 3,4 between $1850 \mathrm{ft}$. and $2340 \mathrm{ft}$. (Fig. 8) and from A 1-81, Core 3 between $3750 \mathrm{ft}$. and $3773 \mathrm{ft}$. These are dominated by the polygonomorph acritarchs, Veryhachium trispinosum and $V$. valiente but also include acanthomorphs such as Diexallophasis and Multiplicisphaeridium. The lack of diagnostic acritarchs of post convolutus zone would indicate the age of the samples as Rhuddanian to Early Aeronian but no higher than convolutus zone. Wells A 1-46, Core 2 between 9710 to $9721 \mathrm{ft}$. (Fig. 8); D 1-31, Core 1, 6105 to $6160 \mathrm{ft}$. (Fig. 8) and F1-46, Core 3, 8852 to $8858 \mathrm{ft}$. all yield rich assemblages dominated by Multiplicisphaeridium, Diexallophasis and Veryhachium and characteristic species of Visbysphaera, Cymbosphaeridium, Oppilatala and ?Dateriocradus. The presence of forms such as Oppilatala eoplanktonica, ?Dateriocradus monterrosae, Multiplicisphaeridium arbusculiferum, Diexallophasis caperoradiola and Visbysphaera gotlandicum indicate the assemblages are of post-convolutus zone age, thus late Aeronian, as an oldest date.

Regional palynological differences in the acritarch assemblages are observed between North Africa and Great Britain, which are consistent with the views of Cramer (1970 - see Silurian references) and Cramer \& Diez (1972). During the Silurian the North African region belonged to one realm (the Neoveryhachium carminae "facies") and Great Britain to another, the Deunffia-Domasia realm. Hence genera such asDeunffia and Domasia which are characteristic in Great Britain of equivalent horizons to some of the Libyan material and which are particularly useful in the biozonation of such strata are absent. The attribution of the Libyan assemblages to precise horizon or horizons by comparison with the type area is thus hampered by such variations.
Neoveryhachium carminae is recorded sporadically in most of the samples under study and is a dominant form in Well C1-44, located in Sirte Basin west of the study area.

\section{CHITINOZOANS}

For a long time, well documented information concerning early Llandovery chitinozoans was lacking while uppermost Llandoverian, Wenlockian and Ludlovian assemblages are well known especially from studies carried out in Scandinavia, U.S.S.R., Belgium, Spain and North Africa. Recently investigations in Canada (Achab, 1981) and Estonia (Nestor, 1976, 1980 a-b) provided new data on Early Llandoverian chitinozoans. Four wells (E1-81, D1-31, A1-81 and A1-46) yielded the Silurian chitinozoan assemblages discussed here. Among the species recorded, several are believed to be new and are kept in open nomenclature. These taxa are: Sphaerochitina sp. A (E1-81, 2270ft; 2250 to $2270 \mathrm{ft}$., and 1968 to $1988 \mathrm{ft}$.), Sphaerochitina sp B. (D1-31, 6105 to $6106 \mathrm{ft}$. and 6159 to $6160 \mathrm{ft}$.), Spinachitina sp. B. (A1-81, 3750 to $3773 \mathrm{ft}$., E1-81, 2250 to $2270 \mathrm{ft}$.) Spinachitina sp. C (A1-81, 3750 to $3773 \mathrm{ft}$.) and Angochitina sp. A. The latter is restricted to one sample (1968 to $1988 \mathrm{ft}$.) in Well E1-81, where it is abundant. Associated with these taxa are better known species such as Conochitina edjelensis elongata, Conochitina armillata, Plectochitina pseudoagglutinans and "Sphaerochitina" vitrea. All these species were described from strata referred to the "middle and late" Llandovery in the Sahara (Taugourdeau, 1963). Most of them are well represented in the four samples of Well D1-31 (6105 to $6106 \mathrm{ft}$., 6120 to $6121 \mathrm{ft}$., 6140 to $6141 \mathrm{ft}$., and 6156 to $6160 \mathrm{ft}$.).

The range of forms belonging to Cyathochitina (C.sp. B, Paris 1981 and $C$. cf. campanulaeformis) are useful for stratigraphical purposes. These taxa do not exceed the early part of the Telychian (sensu Cocks et al. 1984). Indeed, they are not yet represented in the uppermost Llandovery outcrops of Gotland (Laufeld, 1974). In addition Cyathochitina occurs in the Juuru (G1-2) and Raikküla (G3) Estonian stages, but is lacking in the Adavere stage (Nestor, 1976). In Anticosti Island (Canada), Cyathochitina s.s. is still present in the Jupiter Formation (Achab, 1981) while in Spain the genus does not reach the uppermost Llandoverian levels of the 


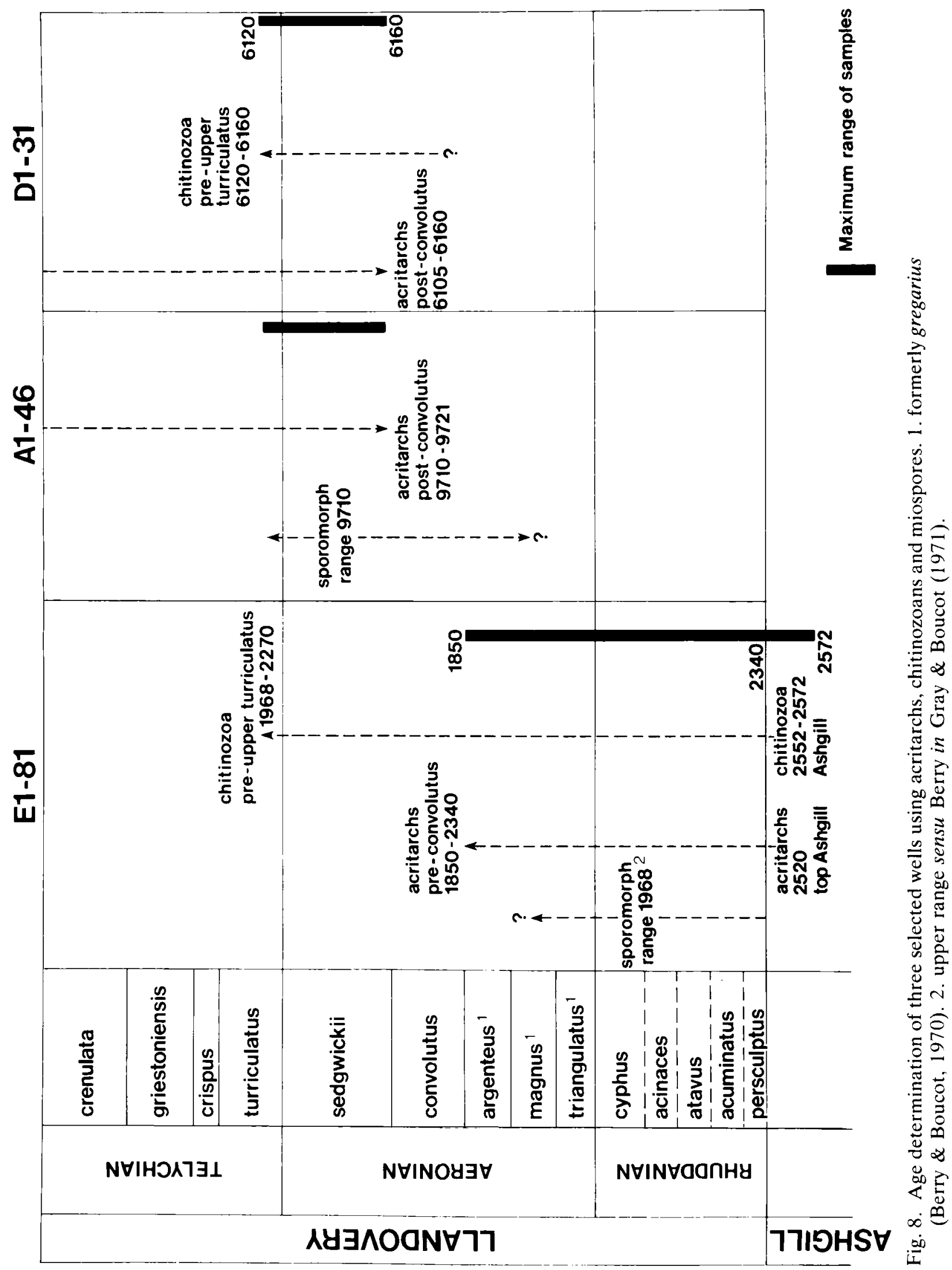


Formigoso Formation (Cramer \& Diez, 1978). From these data it seems likely that the Silurian samples from Wells E1-81, A1-81 and D1-31 (except in D1-31,6105 to $6106 \mathrm{ft}$., where Cyathochitina was not recorded) are not younger than the early Telychian. In addition, in Well D1-31 the occurrence of a few individuals of Conochitina proboscifera and C. (Densochitina) densa is noted, both species are well represented in the uppermost Llandovery and early Wenlock of Gotland (Laufeld, 1974).

On the basis of chitinozoans, the age assignment of level $9710 \mathrm{ft}$. in Well A1-46 is more difficult to establish. Indeed the individuals are rare and the occurrence of a form, closely related to Margachitina leonensis from the Pridoli of Spain (Cramer, 1964), in association with Early Silurian taxa ( $P$. deichaii and $C$. edjelensis elongata, and a form quite similar to $P$. spongiosa), is still unexplained, even though a late Llandovery age is expected for this assemblage.

\section{MIOSPORES}

Silurian miospores have been obtained from core material of two wells, E1-81, (1968 to $1973 \mathrm{ft}$.) and A1-46 (9710 to $9721 \mathrm{ft}$.). The assemblages from the two wells are distinct although showing some features in common. The older of the two miospore assemblages (Well E1-81) consists entirely of dyads and tetrads with some possible alete spores. Dyads e.g. Dyadospora murusdensa, and 'permanent' tetrads, Nodospora sp., are the most common. Some of the dyads are surrounded completely by a diaphanous sheath. In these respects, and in the absence of single grain trilete miospores (i.e. those separated from tetrads), the Well E1-81 assemblages resemble those from the Medina Group (Rhuddanian, early Llandovery) of the Niagara Gorge (Miller \& Eames, 1982). However, the North African assemblage contains occasional specimens of "loose" tetrads, which may suggest a younger age, but is otherwise less diverse than the Niagara Gorge assemblages. The provisional age for this level is early Llandovery and probably Rhuddanian. The basis for this age is partly the close similarities with the Rhuddanian assemblages from Niagara Gorge. In addition Hoffmeister's (1959) Libyan assemblages containing Ambitisporites were dated on graptolites as early to middle Llandovery and the Well E1-81 assemblage is therefore probably older but few well-dated spore assemblages have been described of this age.

A more varied and younger assemblage occurs in the sample from Well A1-46 (9710 to $9721 \mathrm{ft}$.). Two species of dyad are present Dyadospora murusdensa and $D$. murusattenuata, associated with "permanent" tetrads Nodosphaera sp., and Rugosphaera sp., and trilete spores Ambitisporites dilutus. The age of this assemblage is post Rhuddanian to earliest Telychian, approximately Aeronian but probably not earliest Aeronian.
This correlation is made on the assumption that the early/middle Llandovery age of Hoffmeister's material (Berry, in Gray and Boucot, 1971) is roughly equivalent to the middle of the magnus zone (early Aeronian).

\section{REFERENCES}

Achab, A. 1981. Biostratigraphie par les chitinozaires de l'Ordovicien supérieur-Silurien inférieur de l'Ile d'Anticosti. In: Lesperance J. (Ed.), Résultats préliminaires. Subcommission on Silurian Stratigraphy, Ordovician-Silurian Boundary Working Group. Field meeting, Anticosti-Gaspé Quebec 1981, vol II: Stratigraphy and Paleontology, 143-157.

Berry, W. B. N. \& Boucot, A. J. 1970. Correlation of North American Silurian rocks. Geol. Soc. America Spec. Paper 102.

Cocks, L. R. M., Woodcock, N. H., Rickards, R. B., Temple, J. T. \& Lane, P. D., 1984. The Llandovery Series of the Type Area. Bull. Br. Mus. nat. Hist. (Geol). 38 (3) 131-182.

Cramer, F. H. 1970. Distribution of selected Silurian Acritarchs. Rev. Esp. Micropaleontol., num. extr. 203 pp.

Cramer, F. H. \& Diez, M. Del C. R. 1972. North American Silurian palynofacies and their spacial arrangement: acritarchs. Palaeontographica, Abt. B, 138, 107-180.

Cramer, F. H. \& Diez, M. Del. C. R. 1978. Iberian Chitinozoans. 1. Introduction and summary of pre-Devonian data. Palinologia, num. ext., 149-201.

Gray, J. \& Boucot, A. J. 1971. Early Silurian spore tetrads from New York: earliest New World evidence for vascular plants? Science, 173, 918-921.

Hill, P. J. 1974. Stratigraphic palynology of acritarchs from the type area of the Llandovery and the Welsh Borderland. Rev. Palaeobot. Palynol., 18, 11-23.

Hoffmeister, W. S. 1959. Lower Silurian plant spores from Libya. Micropaleontology, 5 (3), 331-334.

Laufeld, S., 1974. Silurian Chitinozoa from Gotland. Fossils and Strata, Oslo., 5, $130 \mathrm{pp}$.

Miller, M. A. \& Eames, L. E., 1982. Palynomorphs from the Silurian Medina Group (Lower Llandovery) of the Niagara Gorge, Lewiston, New York, U.S.A. Palynology, 6, 221254.

Nestor, V., 1976. A microplankton correlation of boring sections of the Raikküla stage, Estonia. Izv. Akad. Nauk. E.S.S.R., Geol., 25, 319-323.

Nestor, V., 1980a. New chitinozoan species from the Lower Llandoverian of Estonia. Izv. Akad. Nauk. E.S.S.R., Geol., 29, 98-107.

Nestor, V., 1980b. Middle Llandoverian Chitinozoans from Estonia. Izv. Akad. Nauk. E.S.S.R., Geol., 29, 136-142.

Paris, F. 1981. Les Chitinozoaires dans le Paléozoique du SudOuest de l'Europe. Mém. Soc. géol. minéral. Bretagne, 26, $498 \mathrm{pp}$.

Taugourdeau, Ph., 1963. Etude de quelques espèces critiques de Chitinozoaires de la region d'Edjelé et compléments à la faune locale. Rev. Micropal., 6 (3), 130-144. 


\section{Explanation of Plate 8}

All figures are $\times 500$

Fig. 1. Saharidia sp. 1 Combaz, 1967. A1-46, Core 2, 9714 ft., Slide 2, P43/3, AGC 79.

Fig. 2. Saharidia sp. 2 Combaz, 1967. F1-46, Core 3, $8852 \mathrm{ft}$., Slide 1, K49, AGC 80.

Fig. 3. Saharidia sp. 2 Combaz, 1967. F1-46, Core 3, $8855 \mathrm{ft}$, Slide 1, E37, AGC 81.

Fig. 4. Tasmanites sp. (Newton) Eisenack, 1958. F1-46, Core 3, $8852 \mathrm{ft}$., Slide 2, D26/2, AGC 82.

Fig. 5. Pterospermella sp. Eisenack, 1972. A1-46, Core 2, $9721 \mathrm{ft}$., Slide 1, G29/4, AGC 83.

Fig. 6. Pterospermella sp. Eisenack, 1972. F1-46, Core 3, 8854 ft., 5 in., Slide 1, K27/4, AGC 84.

Fig. 7. ?Leiosphaeridia sp. (Eisenack) Downie \& Sarjeant, 1963. D1-31, Core 1,6105-6106ft., S. G. 6105-6106/39, AGC 85.

Fig. 8. Leiosphaeridia sp. (Eisenack) Downie \& Sarjeant, 1963. A1-46, Core 2, 9721 ft., Slide 2, S42/4, AGC 86.

Fig. 9. Leiosphaeridia sp. (Eisenack) Downie \& Sarjeant, 1963. A1-46, Core 2, $9721 \mathrm{ft}$., Slide 1, Q32/2, AGC 87.

Fig. 10. Leiosphaeridia wenlockia Downie, 1959. F1-46, Core 3, $8855 \mathrm{ft} .5 \mathrm{in}$., Slide 1, E41, AGC 88.

Fig. 11. Leiosphaeridia wenlockia Downie, 1959. E1-81, Core 3, 1968-1988ft., Slide 1, N40/4, AGC 89.

Fig. 12. Lophosphaeridium parverarum Stockmans \& Willière, 1963. E1-81, Core 4, 2270ft., Slide 1, L45/3, Cluster, AGC 90. 
P. J. Hill

\section{Llandovery Acritarchs}
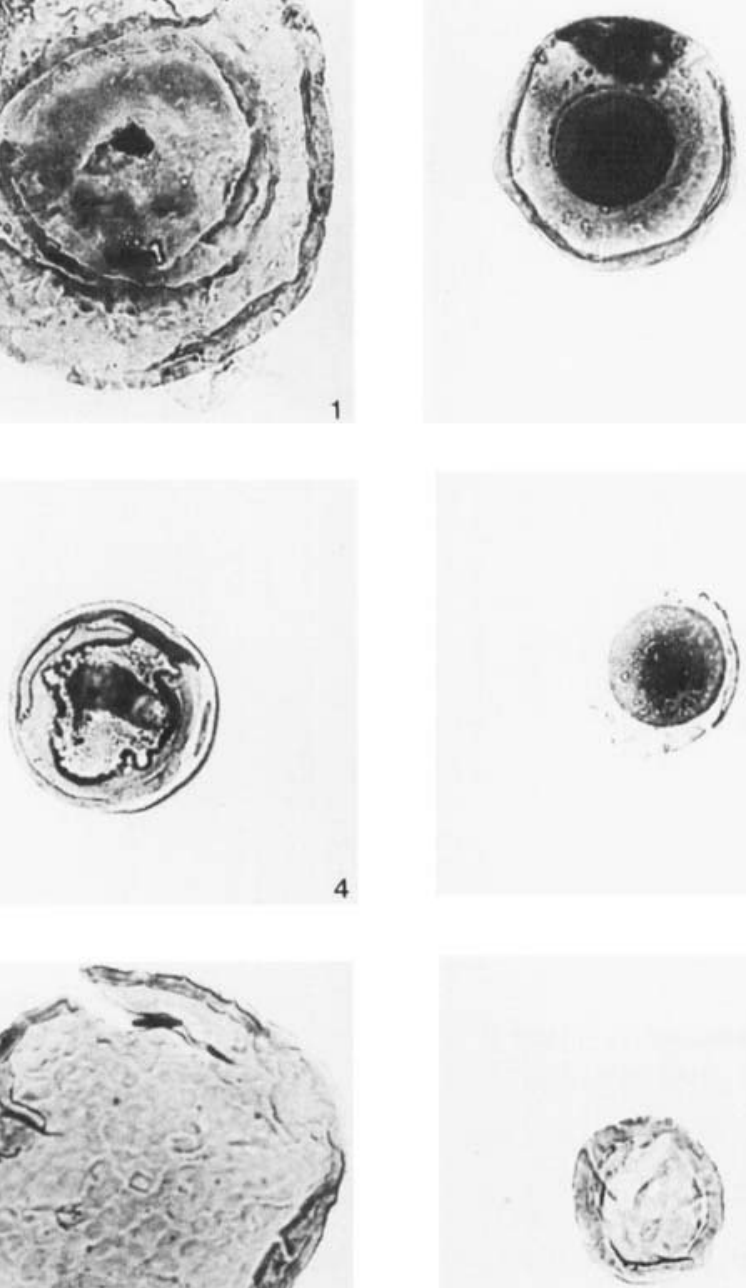

7
Plate 8

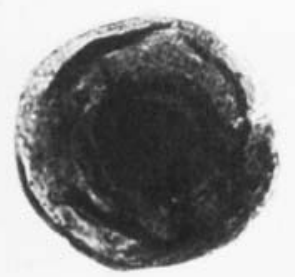

3
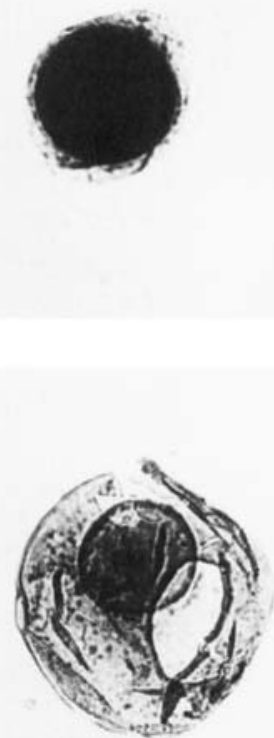

8

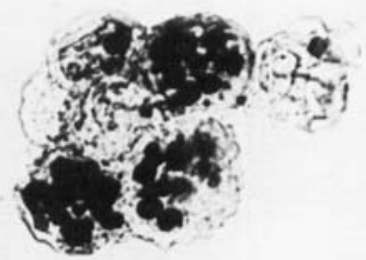




\section{Explanation of Plate 9}

All figures are $\times 500$

Fig. 1. Eupoikilofusa striatifera (Cramer) Cramer, 1970. F1-46, Core 3, $8854 \mathrm{ft}$. 5 in., Slide 1. G42/3, AGC 91.

Fig. 2. Eupoikilofusa striatifera (Cramer) Cramer, 1970. F1-46, Core 3, 8854 ft. 5 in., Slide 1. R42, AGC 92.

Fig. 3. Leiofusa tumida Downie, 1959. F1-46, Core 3, $8854 \mathrm{ft} .5 \mathrm{in}$., Slide 1. R29, AGC 93.

Fig. 4. Leiofusa banderillae Cramer, 1964. F1-46, Core 3, 8852ft. Slide 1. S45/3, AGC 94.

Fig. 5. Leiofusa banderillae Cramer, 1964. F1-46, Core 3, $8852 \mathrm{ft}$. Slide 1. D26, AGC 95.

Fig. 6. Leiofusa fusiformis (Eisenack) Eisenack, 1938. D1-31, Core 1, 6105-6106ft., Slide 1, F47, AGC 96.

Fig. 7. Veryhachium wenlockium Formgroup Downie, 1959. F1-46, Core 3, $8852 \mathrm{ft}$., Slide 1, M40/4, AGC 97.

Fig. 8. Veryhachium trispinosum Formgroup (Eisenack) Cramer, 1964. J1-81A, 12800-12850ft., S.G. 1280012850/35, AGC 98. (Ordovician specimen).

Fig. 9. Veryhachium valiente Cramer, 1964. D1-31, Core 1, 6140-6141ft., Slide 1, M57, AGC 99.

Fig. 10. Neoveryhachium carminae (Cramer) Cramer, 1970. F1-46, Core 3, 8852ft., Slide 1, 030/3, AGC 100.

Fig. 11. ?Dateriocradus monterrosae (Cramer) Dorning, 1981. F1-46, Core 3, 8855ft., Slide 1, M33/1, AGC 101.

Fig. 12. Dictyotidium dictyotum (Eisenack) Eisenack, 1955. F1-46, Core 3, 8852ft., Slide 1, L41/1, AGC 102.

Fig. 13. Buedingiisphaeridium sp. D1-31, Core 1, 6140-6141 ft., Slide 1, K57, AGC 103.

Fig. 14. Tunisphaeridium parvum Deunff \& Evitt, 1968. D1-31, Core 1, 6140-6141ft., Slide 1, K45/1, AGC 104. 
P. J. Hill

Llandovery Acritarchs
Plate 9

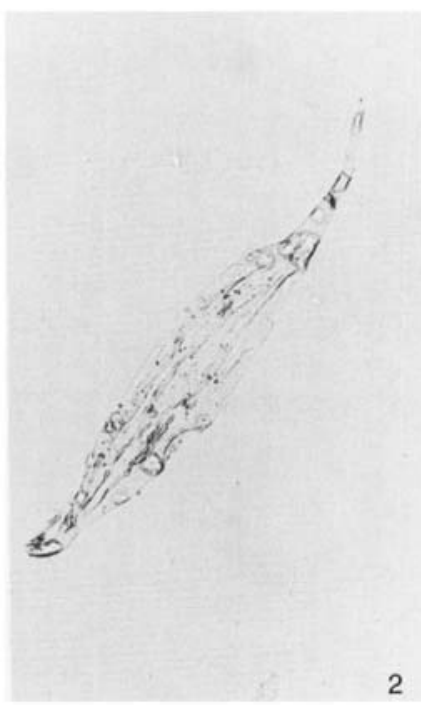

3

4
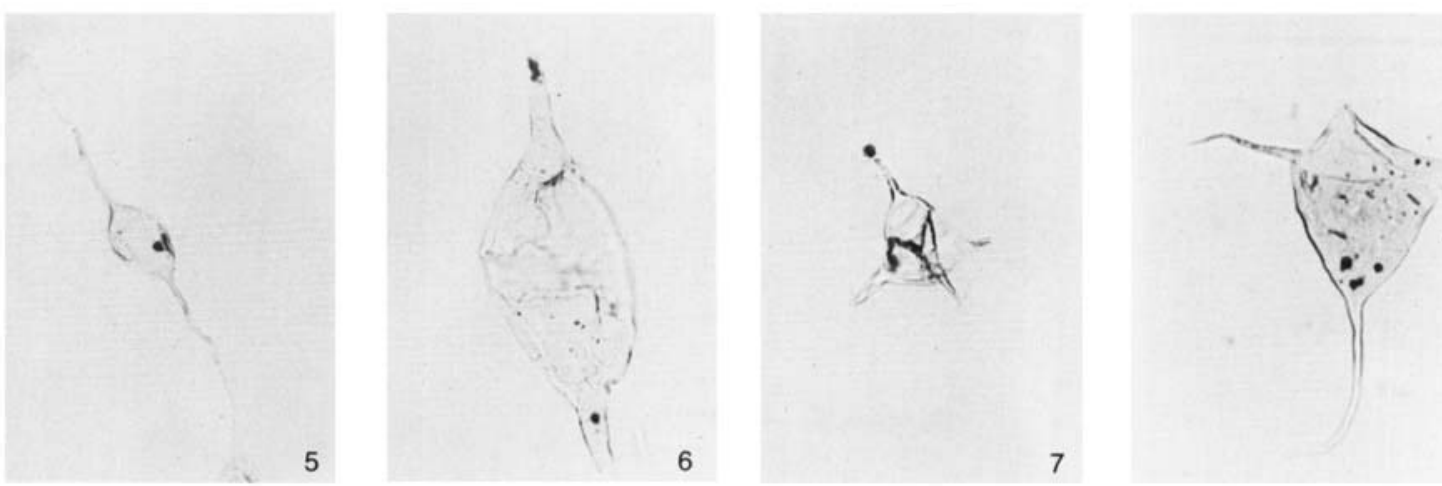

7

8

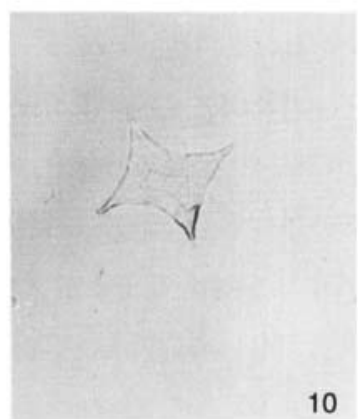

10

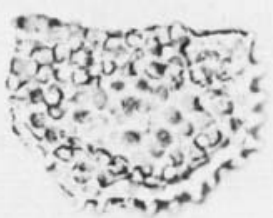

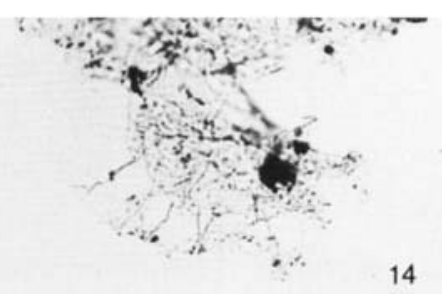




\section{Explanation of Plate 10}

All figures are $\times 500$

Fig. 1. Cymbosphaeridium pilaris (Cramer) Lister, 1970. F1-46, Core 3, $8854 \mathrm{ft} .5$ in., Slide 1, J36/4, AGC 105.

Fig. 2. Oppilatala eoplanktonica Loeblich \& Wicander, 1976. F1-46, Core 3, $8858 \mathrm{ft}$., Slide 1, H39/1, AGC 106.

Fig. 3. Oppilatala eoplanktonica Loeblich \& Wicander, 1976. F1-46, Core 3, $8854 \mathrm{ft}$. 5 in., Slide 1, E24, AGC 107.

Fig. 4. Oppilatala eoplanktonica Loeblich \& Wicander, 1976. F1-46, Core 3, 8852ft., Slide 1, E42, AGC 108.

Fig. 5. Oppilatala eoplanktonica Loeblich \& Wicander, 1976. D1-31, Core 3, 6159-6160ft., Slide 1, G47/2, AGC 109.

Fig. 6. Multiplicisphaeridium fisherii (Cramer) Lister, 1970. F1-46, Core 3, $8855 \mathrm{ft}$., Slide 1, K44, AGC 110.

Fig. 7. Multiplicisphaeridium fisherii (Cramer) Lister, 1970. F1-46, Core 3, 8855ft., Slide 1, T35/3, AGC 111.

Fig. 8. Multiplicisphaeridium ?fisherii (Cramer) Lister, 1970. F1-46, Core 3, 8852ft., Slide 1, H36/1, AGC 112.

Fig. 9. Multiplicisphaeridium fisherii (Cramer) Lister, 1970. F1-46, Core 3, 8852 ft., Slide 1, H39, AGC 113.

Fig. 10. Multiplicisphaeridium arbusculiferum (Downie) Staplin, Jansonius \& Pocock, 1965. F1-46, Core 3, $8858 \mathrm{ft}$., Slide 1, H46, AGC 114.

Fig. 11. Multiplicisphaeridium ramusculosum (Deflandre) Lister, 1970. F1-46, Core 3, 8858ft., Slide 1, F47/3, AGC 115.

Fig. 12. Multiplicisphaeridium ramusculosum (Deflandre) Lister, 1970. D1-31, Core 1, 6105-6106ft., Slide 1, J51/4, AGC 116. 
P. J. Hill

Llandovery Acritarchs

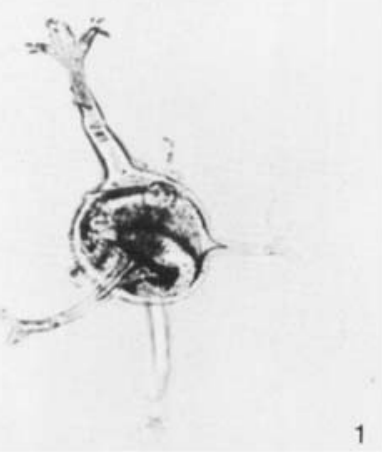

Plate 10
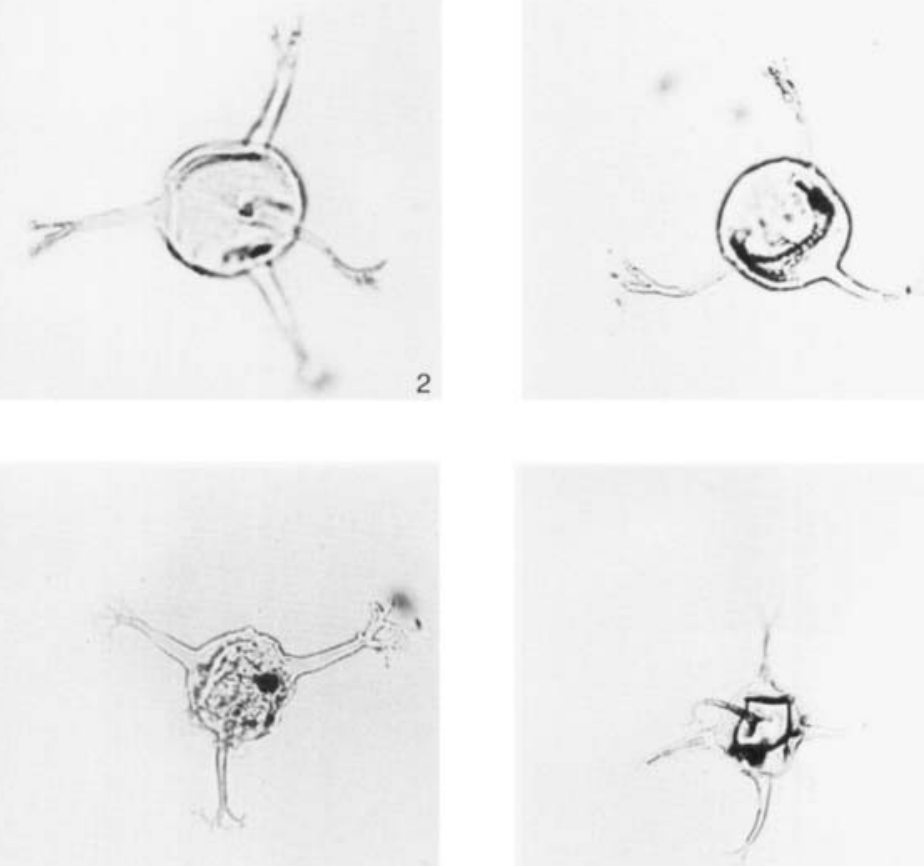

6

9

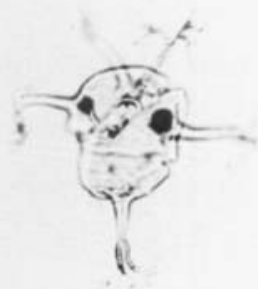




\section{Explanation of Plate 11}

All figures are $\times 500$

Fig. 1. Diexallophasis denticulata (Stockmans \& Willière) Loeblich 1969. F1-46, Core 3, 8855ft., Slide 1, E39, AGC 117.

Fig. 2. Diexallophasis denticulata (Stockmans \& Willière) Loeblich, 1969. D1-31, Core 1,6140-6141 ft., Slide 1, H46/4, AGC 118.

Fig. 3. Diexallophasis caperoradiola Loeblich 1969. F1-46, Core 3, 8854ft. 5 in., Slide 1, L31/1, AGC 119.

Fig. 4. Diexallophasis caperoradiola Loeblich 1969. F1-46, Core 3, 8852ft., Slide 1, G34/4, AGC 120.

Fig. 5. Diexallophasis denticulata (Stockmans \& Willière) Loeblich 1969. F1-46, Core 3, $8852 \mathrm{ft}$., Slide 1, 039/4, AGC 121.

Fig. 6. Diexallophasis caperoradiola Loeblich 1969. D1-31, Core 1, 6159-6160ft., Slide 1, 052, AGC 122.

Fig. 7. Visbysphaera microspinosa (Eisenack) Lister 1970. F1-46, Core 3, 8852ft., Slide 1, D29/4, AGC 123.

Fig. 8. Visbysphaera microspinosa (Eisenack) Lister 1970. F1-46, Core 3, 8852ft., Slide 1, G34/3, AGC 124.

Fig. 9. Visbysphaera microspinosa (Eisenack) Lister 1970. F1-46, Core 3, 8852ft., Slide 1, Q35/3, AGC 125.

Fig. 10. Visbysphaera gotlandicum (Eisenack) Lister 1970. D1-31, Core 1,6159-6160ft., Slide 1, M42/1, AGC 126.

Fig. 11. Visbysphaera microspinosa (Eisenack) Lister 1970. F1-46, Core 3, 8852 ft., Slide 1, Q34/4, AGC 127.

Fig. 12. ?Tylotopalla sp. Loeblich, 1969. A1-46, Core 2, $9710 \mathrm{ft}$., Slide 2, N50/3, AGC 128. 
P. J. Hill

Llandovery Acritarchs
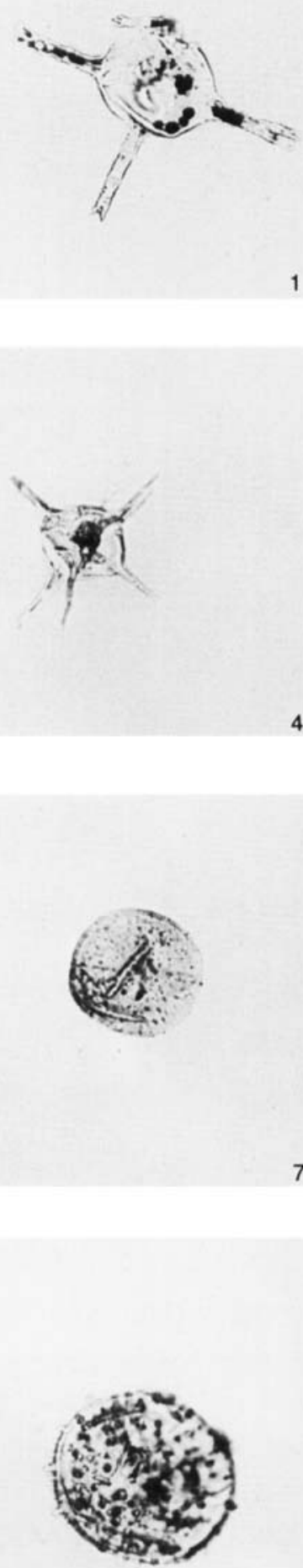

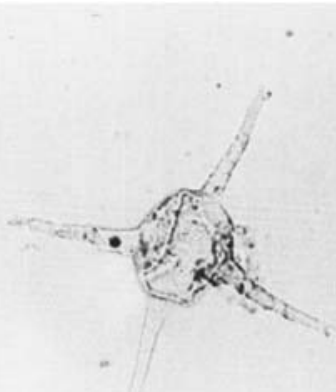

2
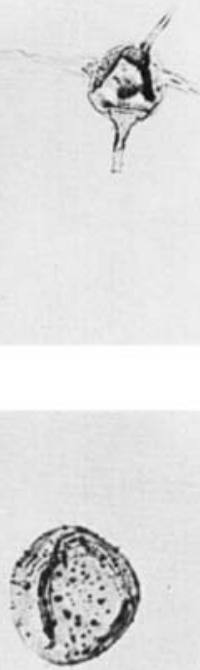

8

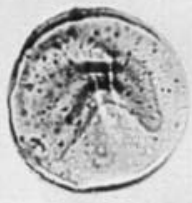

11
Plate 11

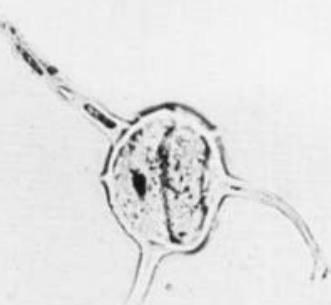

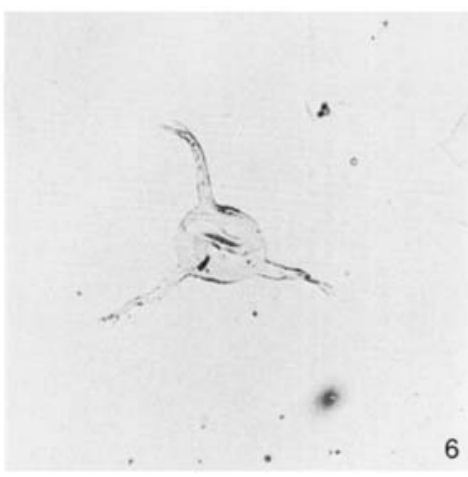

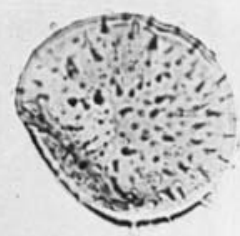

2 


\section{Explanation of Plate 12}

Fig. 1. Plectochitina sp. aff. sylvanica (Jenkins, 1970). A1-81, 3750-3773 ft., Slide 8, 032, $\times 300$, AGC 129. (P. sylvanica is an Ashgillian species).

Fig. 2. Spinachitina sp. B. A1-81, 3750-3773ft., Slide 8, 036, ×300, AGC 130.

Fig. 3. Spinachitina sp. B. A1-81, 3750-3773ft., Slide 8, Q37/2, ×300, AGC 131.

Fig. 4 a-b. Belonechitina postrobusta ?(Nestor, 1980a). A1-81.3750-3773ft., Slide 8, Q37/1, 4a: $\times 300 ; 4 \mathrm{~b}: \times 1000$, AGC 132. ( $B$. postrobusta is restricted to the Early Llandovery).

Fig. 5 a-b. Plectochitina pseudoagglutinans (Taugourdeau, 1963). A1-81.3750-3773ft., Slide 8, 038, 5a: $\times 300 ; 5$ b: $\times 1000$, AGC 133. ("Middle-Upper" Llandovery).

Fig. 6. Spinachitina sp. C. A1-81, 3750-3773ft., Slide 8, P37/3, ×350, AGC 134.

Fig. 7 a-b. Spinachitina sp. B. A1-81, 3750-3773ft., Slide 8, P34/2, 7a: × 300; 7b: × 1500, AGC 135.

Fig. 8. Ancyrochitina laevaensis Nestor, 1980a. A1-81, 3750-3773ft., Slide 8, P32, $\times 400$, AGC 136. (Earliest Llandovery).

Fig. 9 a-b. Spinachitina sp. B. E1-81, 2250-2270ft., Slide 8, P40/3, 9a: ×300; 9b: × 1000, AGC 137. (Early Llandovery).

Fig. 10a-b. Sphaerochitina sp. A. E1-81, 2250-2270ft., Slide 6, P40/4, 10a: ×300; 10b: × 1250, AGC 138. (Early Llandovery).

Fig. 11. Cyathochitina sp. B. Paris, 1981. (=C. kuckerciana Eisenack in Achab, 1981 pl. 4, fig. 15). E1-81, 2250$2270 \mathrm{ft} .$, Slide 6, N39, × 200, AGC 139. 
F. Paris

Plate 12

Llandovery Chitinozoans
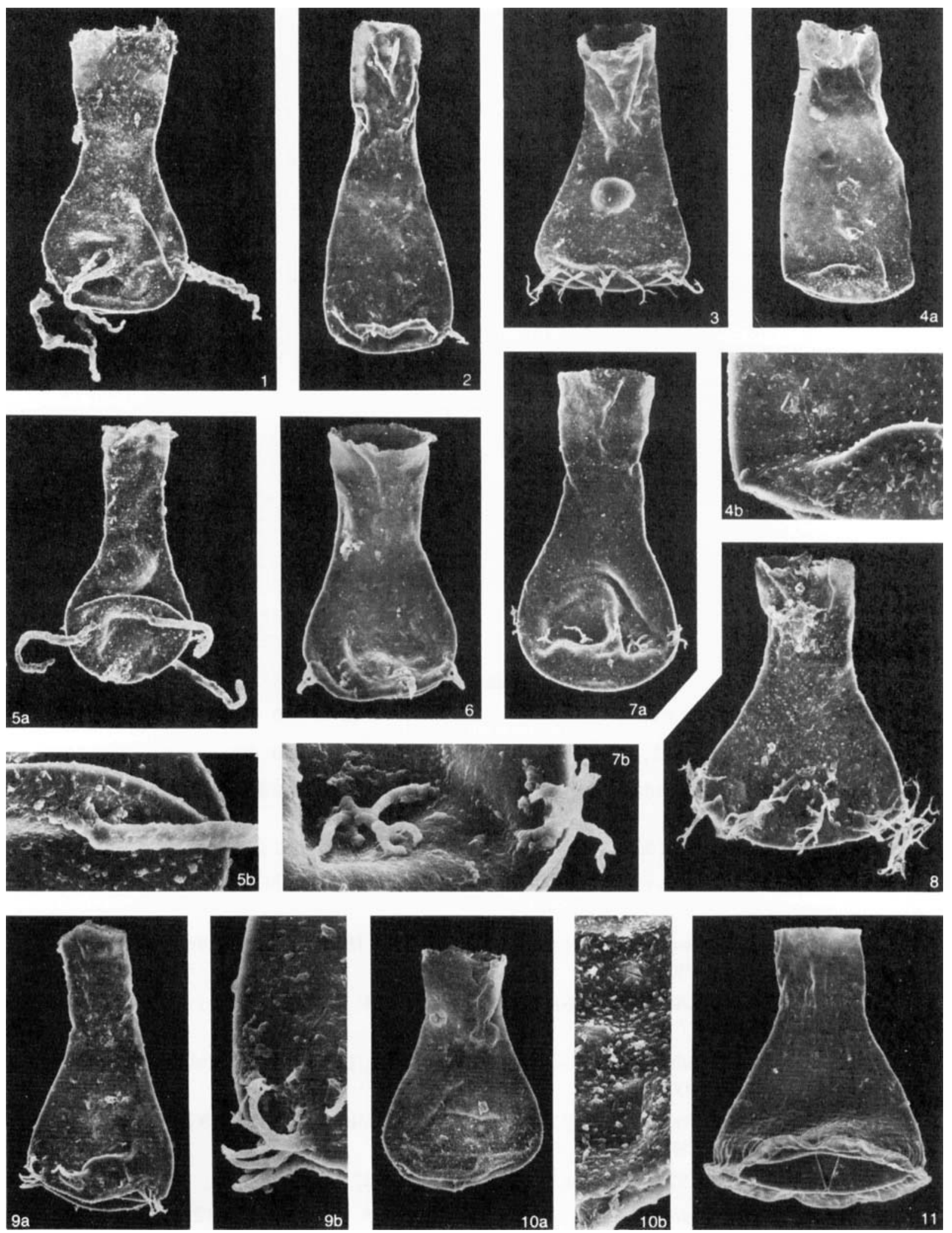


\section{Explanation of Plate 13}

Fig. 1a-b. Angochitina sp. A. E1-81, 1968-1988ft., Slide 7, S37/1, 1a: ×250; 1b: $\times 2000$, AGC 140 .

Fig. 2a-b. Angochitina sp. A. E1-81, 1968-1988ft., Slide 7, 034/3, 2a: × 250; 2b: × 1500, AGC 141.

Fig. 3. Ancyrochitina ancyrea (Eisenack, 1931). E1-81, 1968-1988ft., Slide 7, M36/3, ×250, AGC 142. (Late Ashgill - Early Lochkovian).

Fig. 4. Angochitina sp. A. E1-81, 1968-1988ft., Slide 7, P34/1, × 250, AGC 143.

Fig. 5. Pterochitina deichaii Taugourdeau, 1963. A1-46, $9710 \mathrm{ft}$., Slide 12, N33/4, ×500, AGC 144. ("MiddleUpper" Llandovery).

Fig. 6. Conochitina edjelensis elongata Taugourdeau, 1963. E1-81, 1968-1988ft., Slide 7, L34, ×250, AGC 145 . ("Middle-Upper" Llandovery).

Fig. 7. Plectochitina spongiosa ?(Achab, 1977b). A1-46, $9710 \mathrm{ft}$., Slide 12, N36, $\times 300$, AGC 146. (P. spongiosa is an Ashgill - Early Llandovery? species).

Fig. 8. Plectochitina pseudoagglutinans (Taugourdeau, 1963). E1-81, 1968-1988ft., Slide 7, K37, × 300, AGC 147. ("Middle-Upper" Llandovery).

Fig. 9a-b. ?Margachitina leonensis (Cramer, 1964). A1-46, $9710 \mathrm{ft}$., Slide 12, N37, 9a: × 300; 9b: × 1250, AGC 148. (M. leonensis is a Pridolian species).

Fig. 10. Plectochitina sp. A-46, $9710 \mathrm{ft}$., Slide 12, N33, ×350, AGC 149.

Fig. 11. Conochitina edjelensis elongata Taugourdeau, 1963. A1-46, $9710 \mathrm{ft}$., Slide 12, N37/2, ×250, AGC 150 . ("Middle-Upper" Llandovery). 


\section{Llandovery Chitinozoans}
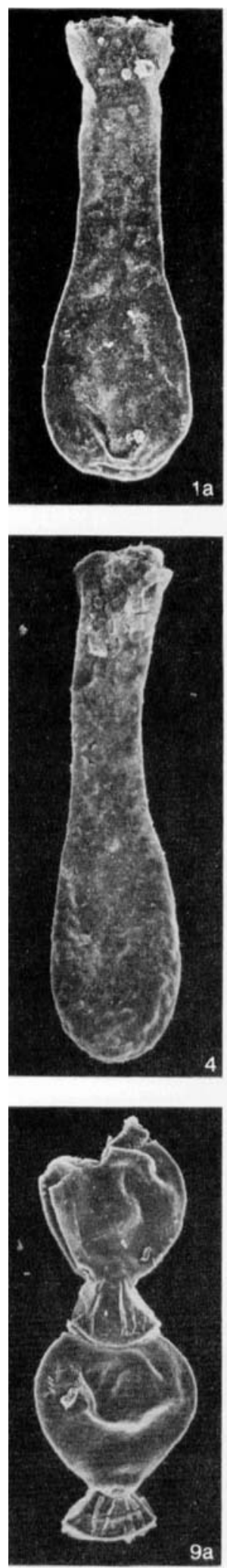
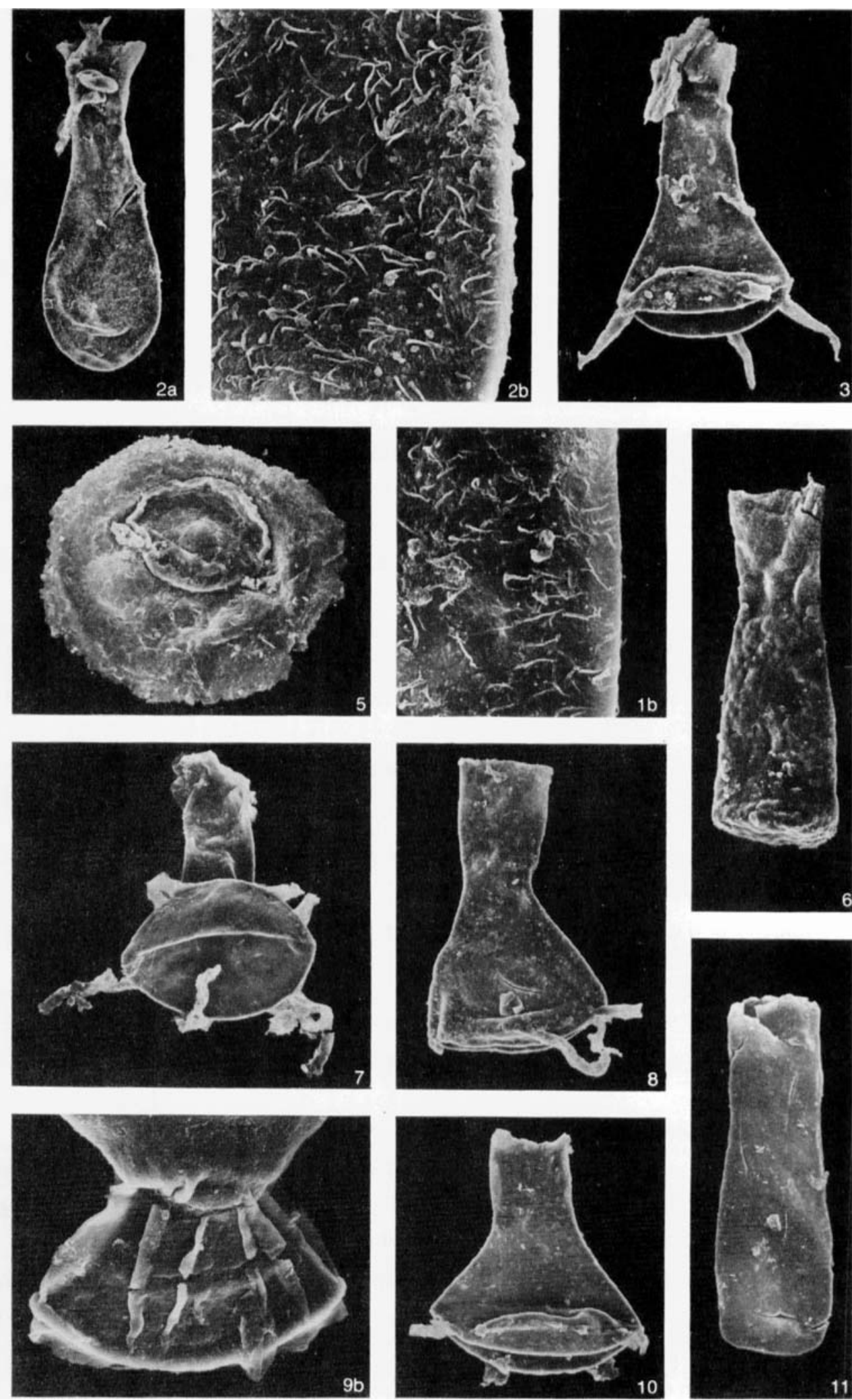
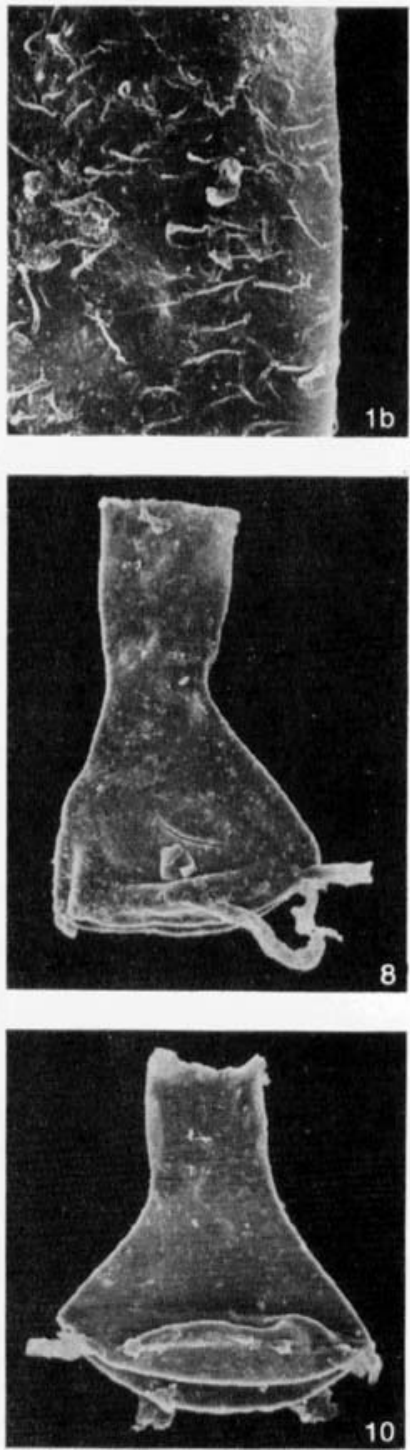
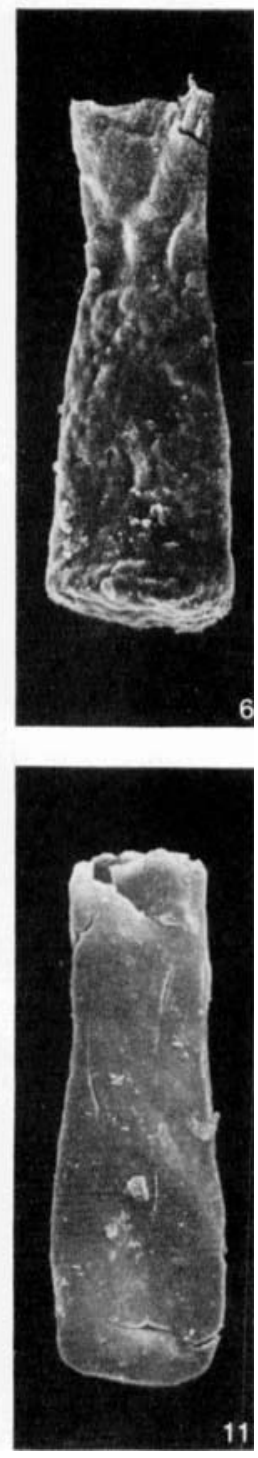


\section{Explanation of Plate 14}

Fig. 1a-b. Conochitina proboscifera Eisenack, 1937. D1-31, 6120-6121ft., Slide 10, P37, 1a: $\times 250 ; 1 \mathrm{~b}: \times 1000$, AGC 151. (Late Llandovery - Early Wenlock).

Fig. 2. Conochitina armillata Taugourdeau \& Jekhowsky, 1960. D1-31, 6159-6160ft., Slide 9, K40/4, ×250, AGC 152. ("Middle-Upper" Llandovery).

Fig. 3. Conochitina armillata Taugourdeau \& Jekhowsky, 1960. D1-31, 6159-6160ft., Slide 9, K40/4, ×250, AGC 153. ("Middle-Upper" Llandovery).

Fig. 4. Ancyrochitina cf. tomentosa Taugourdeau \& Jekhowsky, 1960. D1-31, 6159-6160ft., Slide 9, N40, ×400, AGC 154. (A. tomentosa ranges from the Wenlock? up to the Lochkovian).

Fig. 5. Cyathochitina cf. campanulaeformis (Eisenack, 1931). (= C. campanulaeformis in Achab, 1981, pl. 5, fig. 18). D1-31, 6159-6160ft., Slide 9, L35/4, $\times 200$, AGC 155. (C. campanulaeformis s.l. ranges from the Llanvirn up to the Llandovery, where it never exceeds the Fronian).

Fig. 6. Pterochitina deichaii Taugourdeau, 1963. D1-31, 6159-6160ft., Slide 9, P36, ×500, AGC 156. ("MiddleUpper" Llandovery).

Fig. 7. Calpichitina (Densochitina) densa (Eisenack, 1962). D1-31, 6120-6121 ft., Slide 10, P37, ×500, AGC 157. (Late Llandovery - Early Wenlock).

Fig. 8a-b. Conochitina armillata Taugourdeau \& Jekhowsky, 1960. D1-31, 6105-6106ft., Slide 11, P33, 8a: $\times 300$; 8b: $\times 1000$, AGC 158. ("Middle-Upper" Llandovery).

Fig. 9a-b. Sphaerochitina sp. A. D1-31, 6159-6160ft., Slide 9, L38/3, 9a: ×300; 9b: ×1000, AGC 159.

Fig. 10. Pterochitina deichaii Taugourdeau, 1963. D1-31,6120-6121 ft., Slide 10, T36, ×500, AGC 160. ("MiddleUpper" Llandovery).

Fig. 11a-b. Sphaerochitina sp. B. D1-31, 6159-6160ft., Slide 9, N36, 11a: $\times 300 ; 11 \mathrm{~b}: \times 1000$, AGC 161 .

Fig. 12. Ancyrochitina onniensis ?Jenkins, 1967. D1-31, 6159-6160ft., Slide 9, N38/3, ×400, AGC 162. (A. onniensis ranges from Late Caradoc up to Ashgill).

Fig. 13. Sphaerochitina sp. A. D1-31, 6159-6160ft., Slide 9, N40/2, ×300, AGC 163.

Fig. 14. Calpichitina (Densochitina) densa (Eisenack, 1962). D1-31, 6159-6160ft., Slide 9, L40/2, × 400, AGC 164. (Late Llandovery - Early Wenlock).

Fig. 15. Ancyrochitina sp. aff. ansarviensis Laufeld, 1974. D1-31,6105-6106ft., Slide 11,030, × 400, AGC 165. (A. ansarviensis is an Early Wenlock species). 
F. Paris

Llandovery Chitinozoans
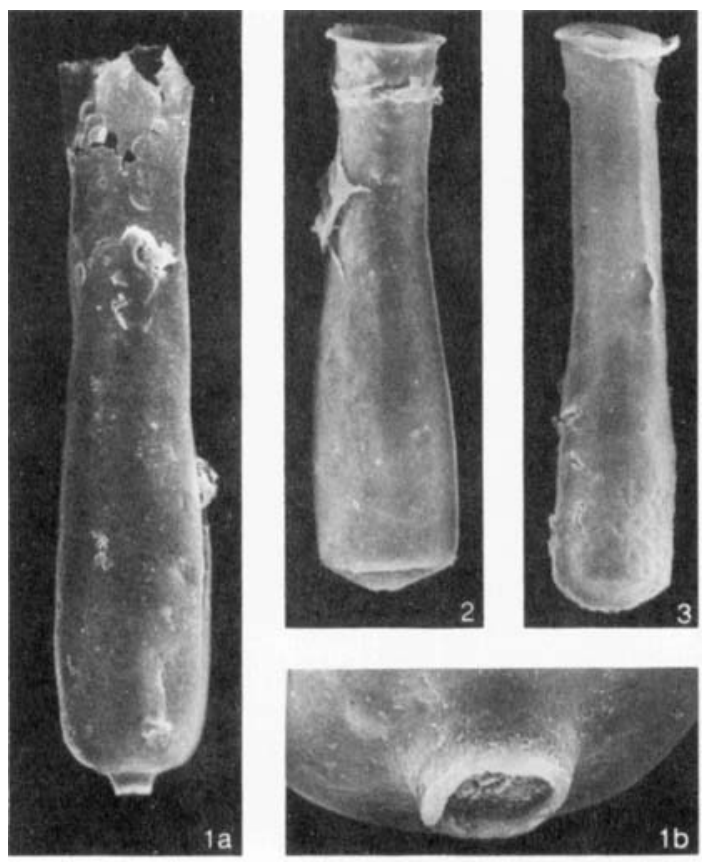

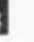
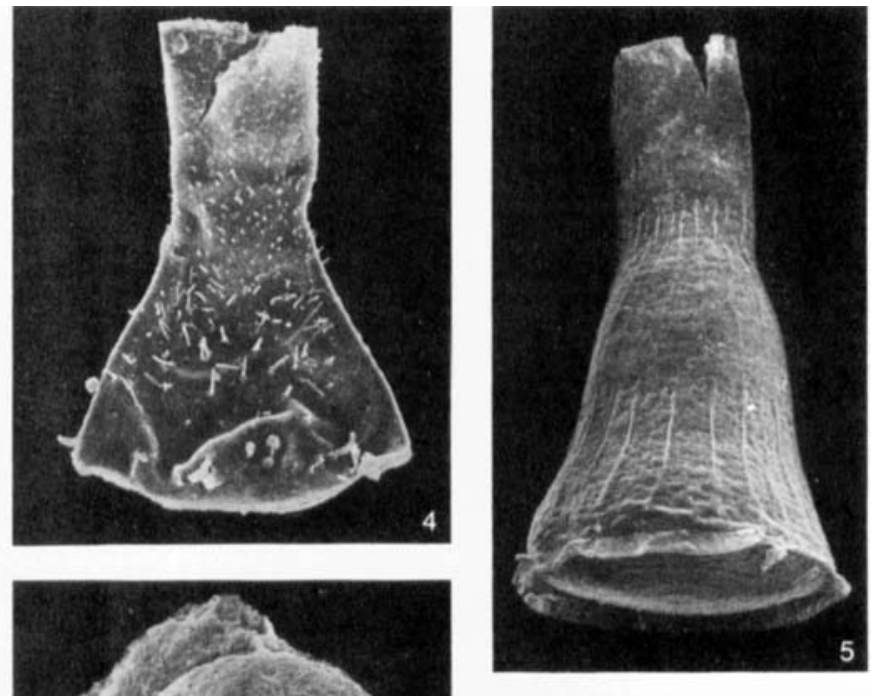

16
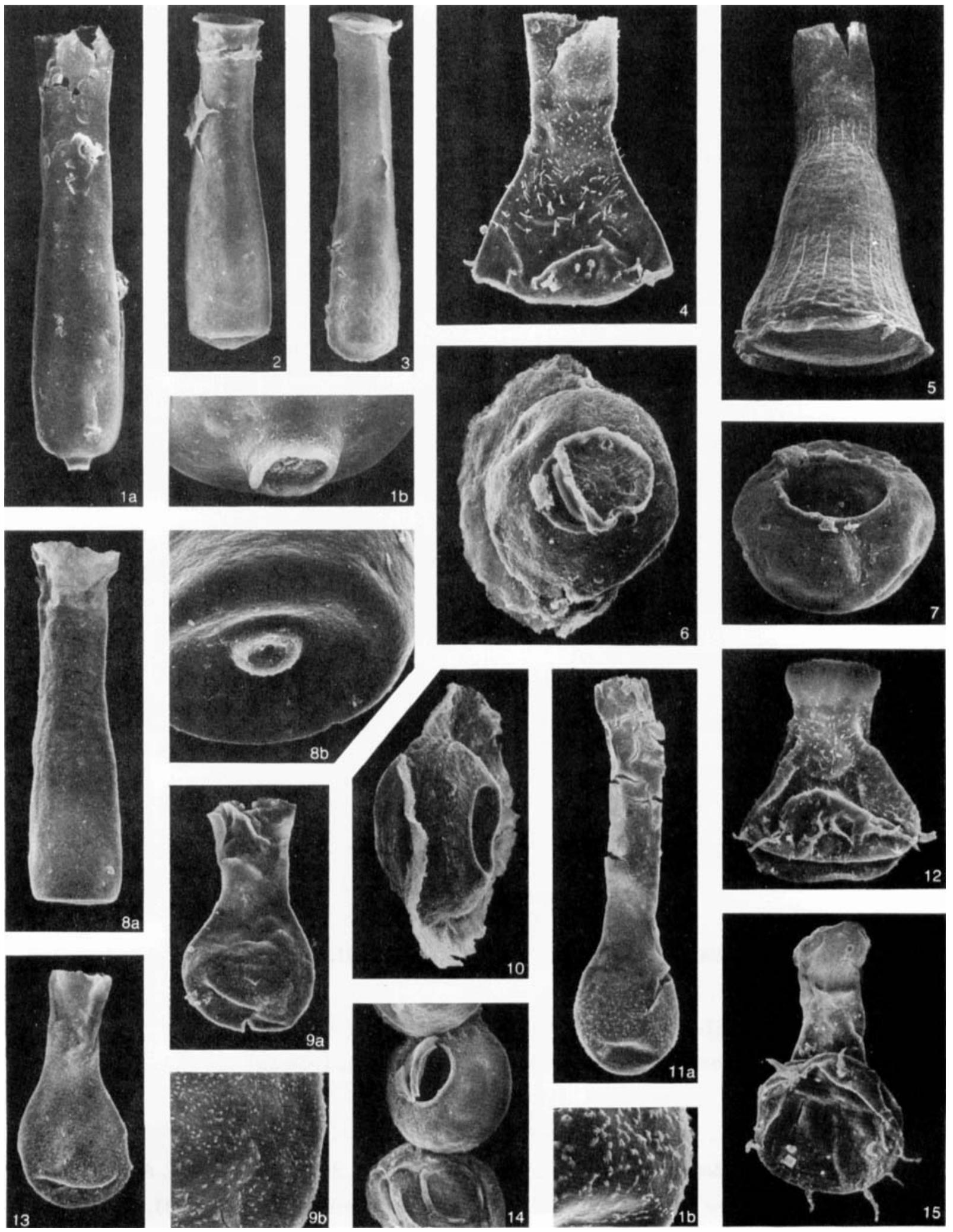

$9 a$
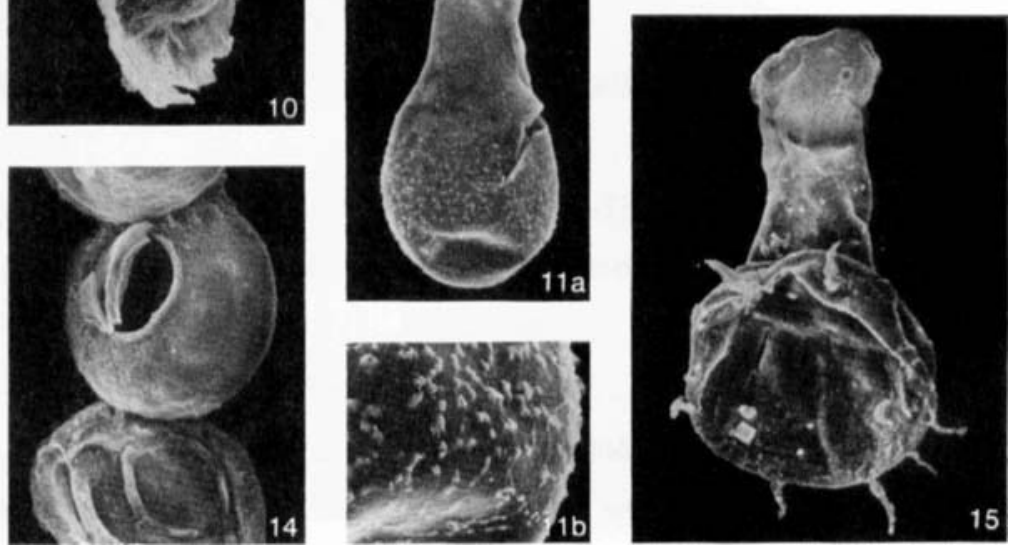


\section{Explanation of Plate 15}

All figures are $\times 1000$

Fig. 1. cf. Tetrahedraletes medinensis Strother \& Traverse, 1979. E1-81, 2520-2550ft., L39/2, AGC 166.

Fig. 2. "Loose" tetrad. E1-81, 1968-1973ft., Q33/4, AGC 167.

Fig. 3. "Permanent" tetrad. E1-81, 2520-2550ft., R33/2, AGC 168.

Fig. 4. cf. Nodospora burnhamensis Strother \& Traverse, 1979. E1-81, 1968-1973ft., P33, AGC 169.

Fig. 5. "Loose" tetrad. E1-81, 2520-2550ft., D40, AGC 170.

Fig. 6. "Loose" tetrad. E1-81, 2520-2550ft., N44/3, AGC 171.

Fig. 7. Dyadospora cf. murusdensa Strother \& Traverse, 1979. E1-81, 2520-2550ft., E35, AGC 172.

Fig. 8. Dyadospora murusdensa Strother \& Traverse, 1979. E1-81, 1968-1973ft., K31-L31, AGC 173.

Fig. 9. Dyadospora murusdensa Strother \& Traverse, 1979. E1-81, 1968-1973ft., 034, AGC 174. 
J. B. Richardson

Llandovery Miospores
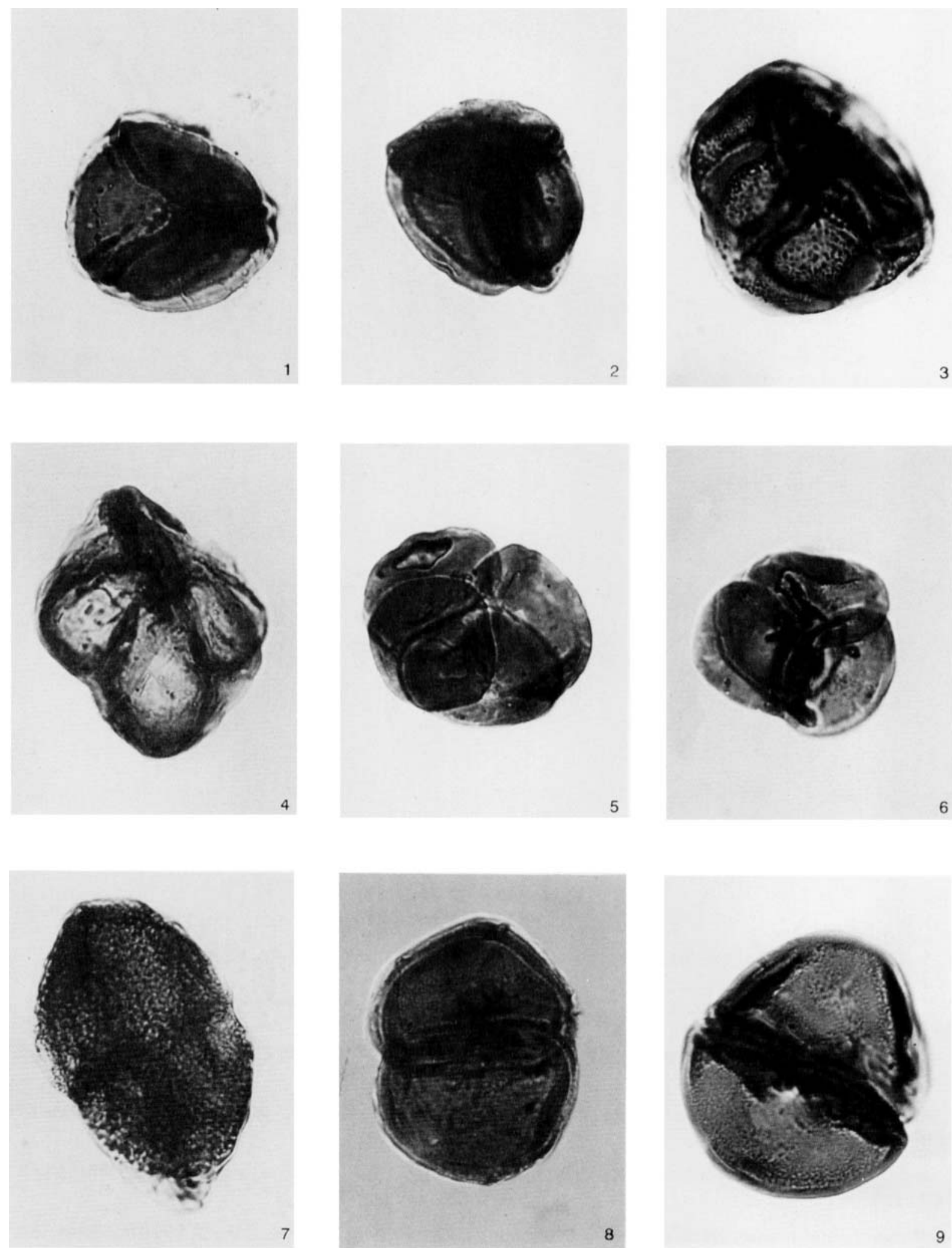

Plate 15

3 


\section{Explanation of Plate 16}

All figures are $\times 1000$

Fig. 1. Archaeozonotriletes cf.chulus var. nanus Richardson \& Lister, 1969. A1-46, Core 2, 9710 ft., Slide 816K, F32, AGC 175.

Fig. 2. Tetrad A1-46, Core 2, $9710 \mathrm{ft}$., Slide 816K, Q43, AGC 176.

Fig. 3. Ambitisporites dilutus (Hoffmeister) Richardson \& Lister, 1969. A1-46, Core 2, $9710 \mathrm{ft}$., Slide 816A, F38/4, AGC 177.

Fig. 4. Archeozonotriletes cf. chulus var. chulus Richardson \& Lister, 1969. A1-46, Core 2, $9710 \mathrm{ft} .$, Slide 816, Q43, AGC 178.

Fig. 5. Ambitisporites dilutus (Hoffmeister) Richardson \& Lister, 1969. A1-46, Core 2, $9710 \mathrm{ft}$., Slide 816K, P41/3, AGC 179.

Fig. 6. Ambitisporites dilutus (Hoffmeister) Richardson \& Lister, 1969. A1-46, Core 2, $9710 \mathrm{ft}$., Slide 816A, R41, AGC 180. 
J. B. Richardson

Plate 16

Llandovery Miospores
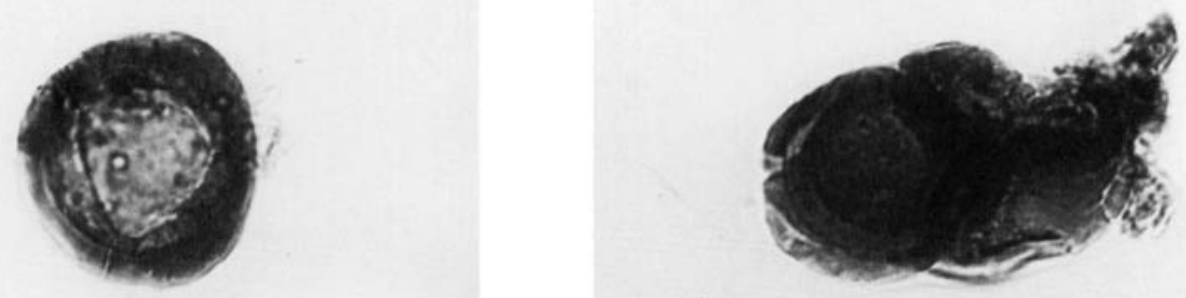

1
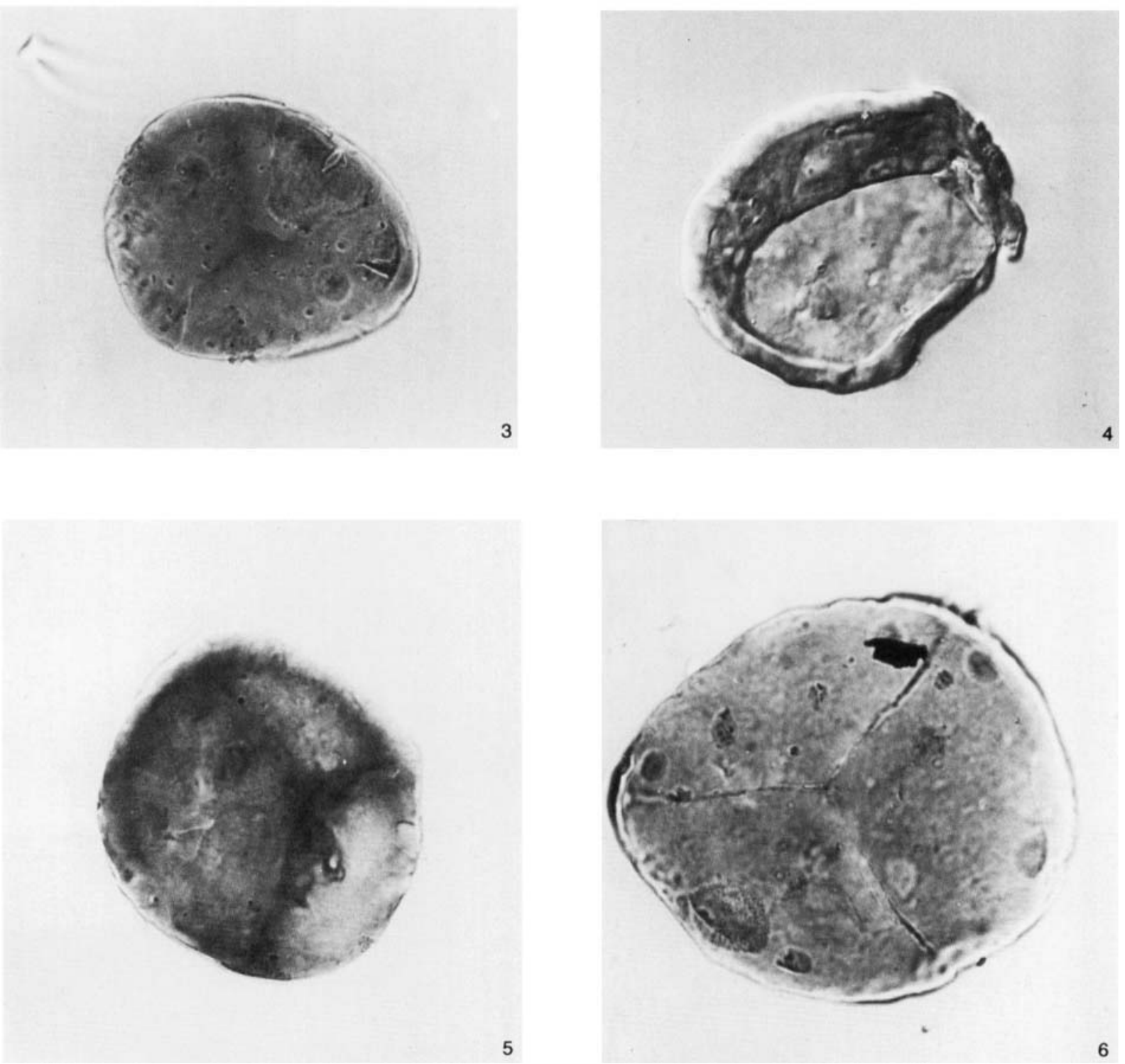